\title{
Spatially resolved X-ray emission of EQ Pegasi
}

\author{
J. Robrade, J.-U. Ness, and J. H. M. M. Schmitt \\ Hamburger Sternwarte, Universität Hamburg, Gojenbergsweg 112, 21029 Hamburg, Germany \\ Received 16 July 2003 / Accepted 23 September 2003

\begin{abstract}
We present an analysis of an XMM-Newton observation of the M dwarf binary EQ Pegasi with a special focus on the spatial structure of the X-ray emission and the analysis of light curves. Making use of data obtained with EPIC (European Photon Imaging Camera) we were for the first time able to spatially resolve the two components in X-rays and to study the light curves of the individual components of the EQ Peg system. During the observation a series of moderate flares was detected, where it was possible to identify the respective flaring component.
\end{abstract}

Key words. stars: binaries: visual - stars: individual: EQ Peg - stars: flare - X-rays: stars

\section{Introduction}

X-ray observations with the Einstein Observatory and ROSAT have shown the ubiquitous occurrence of coronae around most classes of stars. ROSAT studies of volume-limited complete samples of cool stars in the immediate solar neighborhood have shown coronal formation around late-type cool dwarf stars with outer convection zones to be universal; all stars investigated with sufficient sensitivity were found to be surrounded by X-ray emitting coronae (Schmitt et al. 1995; Schmitt \& Liefke 2003). Interestingly, fully convective $M$ dwarfs have also been found to be very active with frequent flares.

EQ Peg is a nearby $(6.25 \mathrm{pc}$ ) visual binary (period $\sim 180 \mathrm{yr}$, separation 5.2") consisting of two $\mathrm{M}$ dwarfs of spectral type M3.5 and M4.5. It was first observed photoelectrically to flare by Roques (1954), and Owen et al. (1972) found both components of the system to be flare stars.

EQ Peg has been observed at radio, optical, EUV, and X-ray wavelengths. Observations in the optical focused on the flare nature of EQ Peg and marked emission line variability during photometric quiescence was found (Bopp 1974) as well as frequent optical flares on both components (Rodonò 1978). A VLA map of EQ Peg at $6 \mathrm{~cm}$ was presented by Topka \& Marsh (1982). They resolved both components and interpreted the radio emission as "quiescent" since they found it unlikely that both components flared at the same time. The radio emission was confined to each component and Topka \& Marsh (1982) concluded that radio production mechanisms do not depend on binary interaction (which is plausible due to the separation of $\sim 25 \mathrm{AU})$.

EQ Peg was observed by all major previous imaging X-ray missions and again found to flare frequently. EQ Peg was observed with the Einstein Observatory (Vaiana et al. 1981) and is

Send offprint requests to: J. Robrade, e-mail: jrobrade@hs.uni-hamburg.de contained in the ESS (Einstein Slew Survey) (Elvis et al. 1992). EXOSAT detected an intense long duration flare during a coordinated observation with the VLA (Pallavicini et al. 1986); a detailed modelling of these flares and the underlying physical properties is presented by Polotto et al. (1988). EQ Peg was also detected in the ROSAT all-sky survey (Hünsch et al. 1999) and rapid flaring was simultaneously observed at optical and X-ray wavelength with MEKASPEC and ROSAT (Katsova et al. 2002), where the source brightened in X-rays by a factor of $\sim 15$. A coordinated VLA, optical, EUVE, and RXTE monitoring of EQ Peg was carried out by Gagné et al. (1998). They found a classic stellar flare with a rapid impulsive phase (radio burst) followed by rapid chromospheric heating and cooling $(U$-band) and more gradual coronal cooling (X-ray and extreme-UV). In addition they found atypical flares with either highly polarized emission with no counterparts at shorter wavelengths or moderately polarized flares that often have shorterwavelength counterparts.

EQ Peg was also observed with XMM-Newton. In Sect. 2 we describe the observations and the methods used for data analysis. Here we focus on the data from the EPIC instruments in order to obtain spatial and temporal information on the two components of EQ Peg. In Sect. 3 we present the results followed by a summary and discussion in Sect. 4.

\section{Observation and data analysis}

EQ Peg A/B ( $V=10.32 \mathrm{mag} / 12.4 \mathrm{mag})$ was observed on 2000 July $9(\mathrm{MJD}=51734)$ with XMM-Newton. The $15 \mathrm{ksec}$ observation of EQ Peg (see Table 1) provided useful data in all EPIC (European Photon Imaging Camera) detectors. The EPIC instrument consists of three CCD cameras with two different types of CCD design, resp. two MOS (Metal Oxide Semi-conductor CCDs) and one PN (pn CCDs), providing imaging and spectroscopy. The EPIC cameras offer the 
Table 1. Observation log of EQ Peg.

\begin{tabular}{lcc}
\hline \hline Instrument (Mode) & Duration (s) & Obs-time \\
MOS (FF, thick F.) & 14600 & 2000-07-09T11:39:13-15:42:32 \\
PN (FF, thick F.) & 12410 & 2000-07-09T12:20:16-15:47:05 \\
\hline
\end{tabular}

possibility to perform extremely sensitive imaging observations over the telescope's field of view of $30^{\prime}$ and in the energy range from 0.15 to $15 \mathrm{keV}$ with good angular and moderate spectral resolution. A detailed description of the XMM instruments can be found in Ehle et al. (2003). All EPIC instruments (MOS/PN) operated in the full frame mode with the thick filter inserted. Unless otherwise indicated we used for our purposes the full energy bandpass of the EPIC instruments, resp. $0.15 \mathrm{keV}$ to $12.0 / 15.0 \mathrm{keV}$.

The data were reduced with the standard XMM-Newton Science Analysis System (SAS) software, version 5.4.1. Light curves and images were produced with standard SAS tools and standard selection criteria were applied for filtering the data. In Fig. 1 we show the image obtained with the MOS 1 detector. The image looks elongated and it is reasonable to assume the elongation is due to emission from both components of EQ Peg. The image elongation can be seen clearly in MOS1, but not in MOS2 and PN due to the triangular shape of the point spread function (PSF) for MOS2 and the bigger pixel size in PN.

For a quantitative analysis of the MOS1 image we developed a fitting procedure applicable to the measured event distribution in order to confirm the detection of the two components of EQ Peg and to determine accurate source positions and count rates. For this procedure we optimize a set of parameters describing the modelled event distribution on the sky plane. The modelled event distribution is constructed on the basis of the PSF (Kirsch 2002), which is composed of a King component plus background

$\mathrm{PSF}=\frac{A}{\left(1+\left(\frac{r}{r_{\mathrm{c}}}\right)^{2}\right)^{\alpha}}+\mathrm{BKG}$

with the core radius $r_{\mathrm{c}}$, the slope $\alpha$ (model parameters), the distance to the peak position $r$, and the amplitude $A$ (source parameters). The background flux BKG was determined from source free regions in the detector and is kept as a fixed model parameter. We place two such King components near the positions of the two sources and apply an optimization algorithm seeking best-fit values for the amplitudes $A_{1}$ and $A_{2}$ and the positions $r_{1}=\left(x_{1}, y_{1}\right)$ and $r_{2}=\left(x_{2}, y_{2}\right)$ of each component. We thus determine the model counts in the spatial bin $(i, j)$

$\boldsymbol{n}_{i, j}=\frac{A_{1}}{\left(1+\left(\frac{r_{1}-r_{i, j}}{r_{\mathrm{c}}}\right)^{2}\right)^{\alpha}}+\frac{A_{2}}{\left(1+\left(\frac{r_{2}-r_{i, j}}{r_{\mathrm{c}}}\right)^{2}\right)^{\alpha}}+\mathrm{BKG}$

and apply Powell's algorithm (Press et al. 1999), a robust multidimensional minimizing routine, in order to minimize the likelihood function

$\mathcal{L}=-2 \sum_{i, j} \log \left(\boldsymbol{n}_{i, j} * \boldsymbol{c}_{i, j}-\boldsymbol{n}_{i, j}\right)$

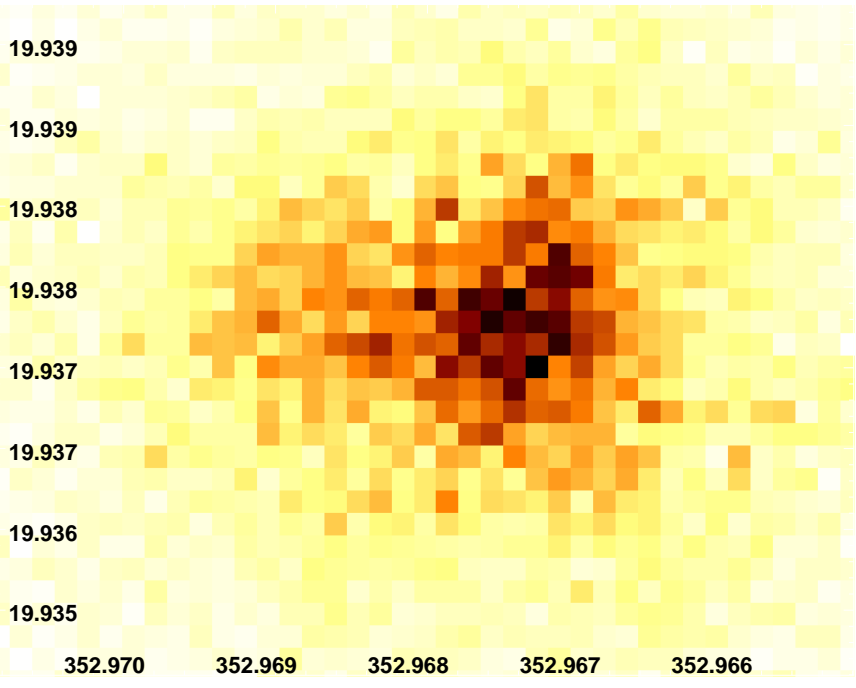

Fig. 1. Image of the EQ Peg system (MOS1) with linear brightness scaling. The image is elongated, suggesting the presence of two components. Analysis shows that the X-ray brighter component is EQ Peg A.

with the measured counts $\boldsymbol{c}_{i, j}$ and model counts $\boldsymbol{n}_{i, j}$. The model counts $\boldsymbol{n}_{i, j}$ are constrained to conserve the total number of counts, i.e., $\sum_{i, j} \boldsymbol{n}_{i, j}=\sum_{i, j} \boldsymbol{c}_{i, j}$.

The mean PSF-parameters $\left(r_{\mathrm{c}}\right.$ and $\alpha$ ) as determined by inflight calibration (Ghizzardi 2001) did not lead to good fit results for our EQ Peg data. It turned out that especially for the value of the core radius $\left(r_{\mathrm{c}}\right)$ binning and pile-up effects have to be considered, while the variation of the slope $\alpha$ is only moderate. In order to find a better representation for the shape of the PSF we carried out a parameter study for our data from EQ Peg and for XMM-Newton observations of single point sources, e.g., $\epsilon$ Eri with a comparable detector configuration and pileup level. From this study we found a slope of $\alpha=1.45$, which agrees with the calibration value, and a value for the core radius of $r_{\mathrm{c}}=4.45$, i.e., a reduction of $\sim 20 \%$, to be better suited to model our data.

With the redetermined PSF parameters we modelled the event distribution. In Fig. 2 we show a one-dimensional representation of our fit results for the EQ Peg observation. For this purpose we integrated along the declination axis, which almost matches the main axis of the EQ Peg system and binned the data into a histogram. As can be seen in Fig. 2, the model describes the data very well.

\section{Results}

\subsection{Determination of exact source positions}

A first inspection of Fig. 1 suggests the presence of two sources. Since the angular resolution of the MOS1 detector is of the same order as the separation of the two components of EQ Peg $\left(\sim 5^{\prime \prime}\right)$, it is clear that we are operating at the limit of the spatial resolving power of the MOS1 detector. We applied our PSF algorithm to the EQ Peg dataset to determine precise source positions. We use the MOS1 data from a $50 \times 50^{\prime \prime}$ field centered on the position of the EQ Peg system. The calculated 


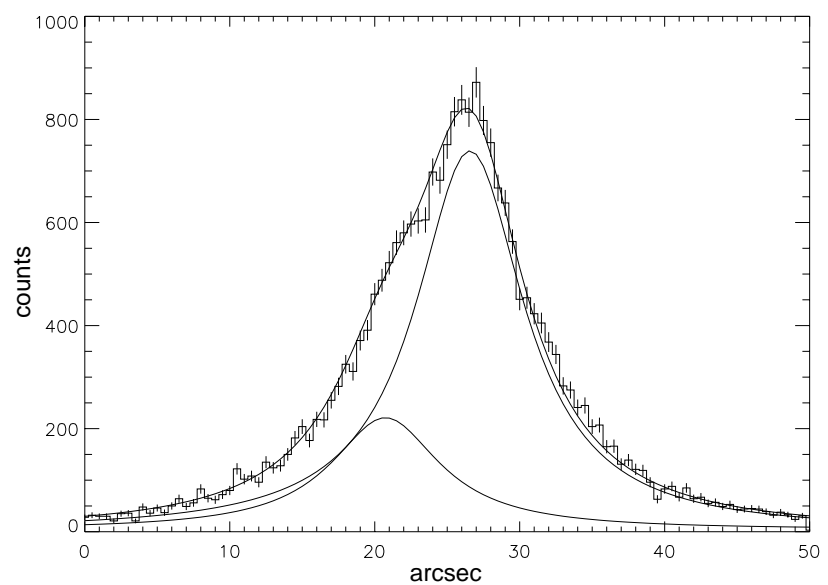

Fig. 2. The event distribution of EQ Peg, fitted with the PSF model. Shown are the individual components as well as the sum of both components compared to the data (histogram).

Table 2. Position fit results (1 $\sigma$ errors), ref. data from Simbad (FK5/2000).

\begin{tabular}{lcc|cc}
\hline \hline & \multicolumn{2}{c}{ EQ Peg A } & \multicolumn{2}{c}{ EQ Peg B } \\
\hline & Fit & Ref. & Fit & Ref. \\
RA (23:31:) & $52.17 \pm 0.01$ & 52.18 & $52.57 \pm 0.02$ & 52.53 \\
& $\left(352.9674^{\circ}\right)$ & & $\left(352.9690^{\circ}\right)$ & \\
Dec (+19:56:) & $14.10 \pm 0.05$ & 14.10 & $14.05 \pm 0.10$ & 13.90 \\
& $\left(19.9373^{\circ}\right)$ & \multicolumn{3}{c}{$\left(19.9372^{\circ}\right)$} \\
\hline EQ Peg System & Fit & \multicolumn{3}{c}{ Ref. } \\
Separation (") & $6.0 \pm 0.3$ & \multicolumn{2}{c}{5.2} \\
\hline Count ratio A/B & $3.4 \pm 0.2$ & $4.2 \pm 0.3$ (quies.) & $2.9 \pm 0.2$ (flare) \\
\hline
\end{tabular}

source positions as listed in Table 2 agree well within the errors with the optical positions taken from literature (Perryman et al. 1997). Here proper motion corrections were not applied since they are small because the observation took place in July 2000. We therefore conclude that we indeed identified the two X-ray sources with the optical counterparts.

The algorithm was also used to determine the absolute number of counts per source and the count ratio of the sources. EQ Peg A was on average a factor of $~ 3.5$ brighter than EQ Peg B during the total observation.

\subsection{Identification of flare activity}

From the image obtained with MOS1 the two components of EQ Peg can be separated. In Fig. 3 we plot the light curves for the EQ Peg system as observed with the different EPIC detectors. Inspection of the total light curves in Fig. 3 shows that the EQ Peg system stayed more or less quiet during the first $8 \mathrm{ksec}$, afterwards a rise in count rate is detected in all three detectors. We therefore divided the data set into two parts separated at $t=7.86 \mathrm{ksec}$ where we consider the first part the quiescent phase and the second part the flaring phase.

In Fig. 4 we display the event distributions of these two subsets; the histograms were created in the same way as in Fig. 2 and are corrected for the different integration times. Here EQ Peg A is the X-ray brighter component on the right, EQ Peg B corresponds to the weaker component on the left

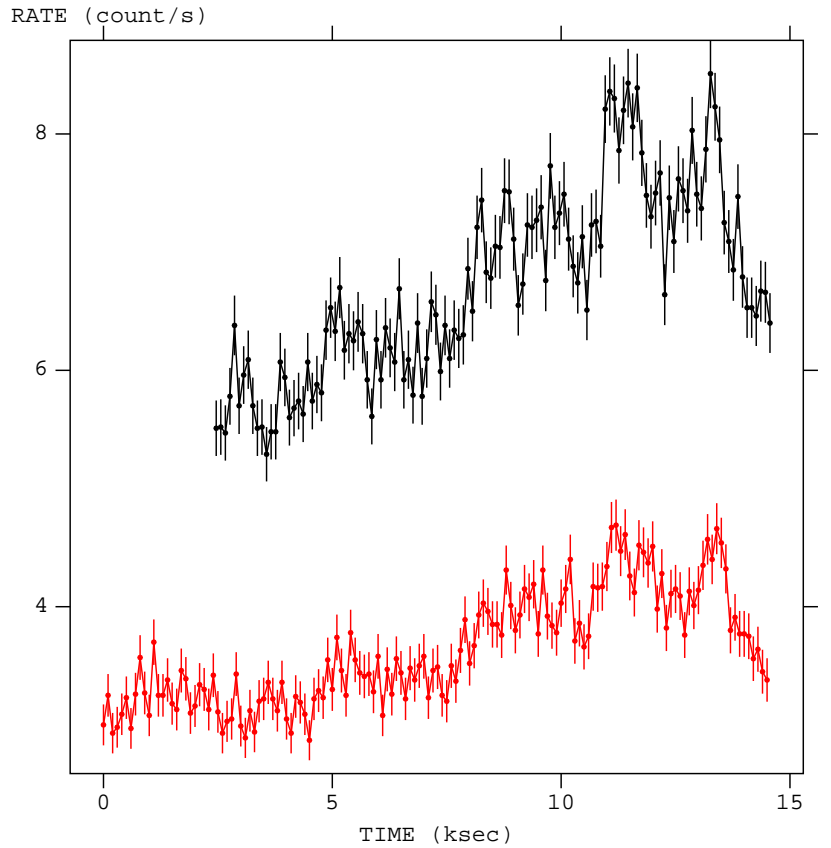

Fig. 3. Light curve of the EQ Peg observation as measured by the PN (black) and MOS1+2 (grey) detectors with $100 \mathrm{~s}$ binning.

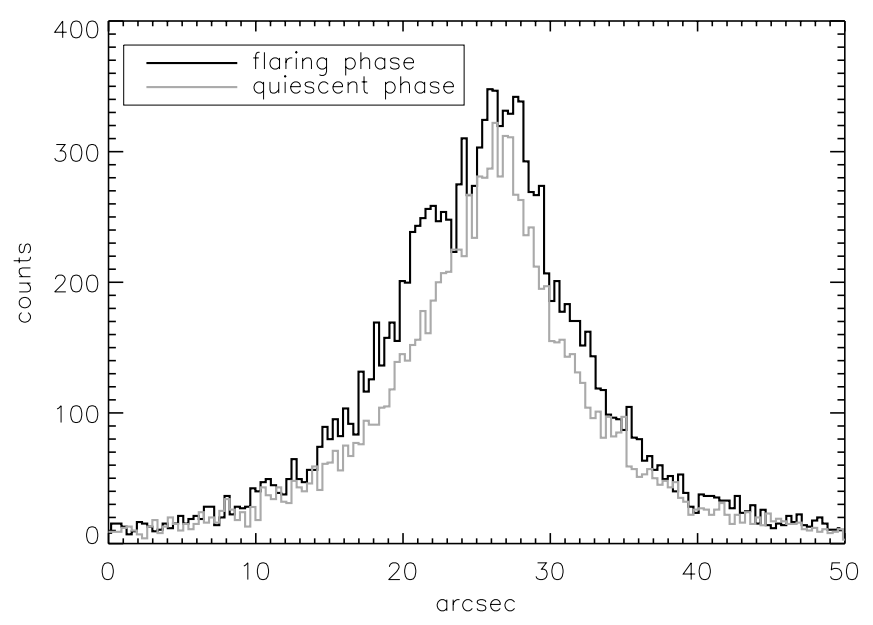

Fig. 4. Comparison of the event distribution during the quiescent and flaring phase.

edge of the event-distribution. In the quiescent phase EQ Peg A dominates the emission and the second component is only marginally visible, while during the flaring phase EQ Peg B brightens up and becomes more clearly visible.

The analysis of the X-ray images as carried out for the total observation (Sect. 3.1) can be repeated for the different phases of activity. Application of our PSF algorithm to the two subsets with variation of only the amplitude parameters returned the count rate of EQ Peg A to be a factor $\gtrsim 4$ higher than for EQ Peg B in the quiescent phase, while in the flaring phase the ratio was $\lesssim 3$. The results of the fitting procedure for the EQ Peg observation are summarized in Table 2. 


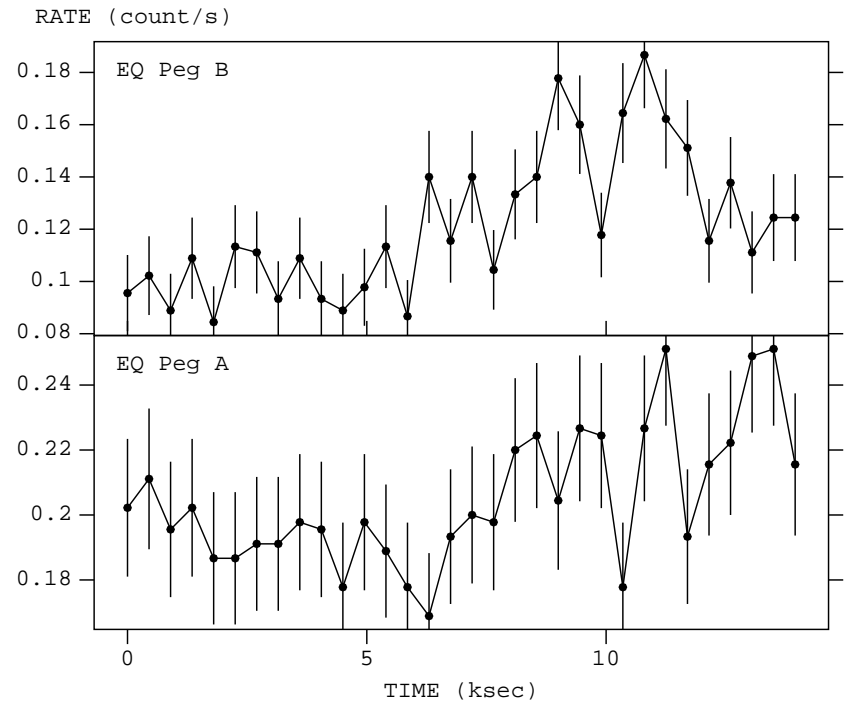

Fig. 5. Light curves of the two components extracted from a circular region around each component.

\subsection{Reconstruction of individual light curves}

Having found that the major flaring activity is due to EQ Peg B, we decided to carry out a systematic light curve analysis of both components of the EQ Peg system. As a first approach we extracted two different light curves from the MOS1 data by placing a circular region with $2.5^{\prime \prime}$ radius around each component, concentrating on the core of the PSF.

In Fig. 5 we show these light curves binned into $7.5 \mathrm{~min}$ bins. Again, EQ Peg B is more variable and its count rate rises by a factor of $\sim 2$ at the peak of the flare while EQ Peg A shows only marginal brightening compared to the quiescent emission level. Clearly, the extraction regions used do contain photon contamination from the respective other component due to the wings of the PSF. Nevertheless, the individual light curves also suggest uncorrelated variability between the A and B components. In particular, the flaring at the end of the observations (e.g., $t \gtrsim 11 \mathrm{ksec}$ ) seems to originate from the A component.

For a more detailed quantitative treatment we utilize our PSF algorithm in order to reconstruct the individual light curves. We divide the dataset into seven time intervals covering the various phases of activity as shown in the upper panel of Fig. 6. From our PSF algorithm a count ratio for each time interval can be determined and in the bottom panel of Fig. 6 we show the development of this count ratio. While the main flaring activity is located on EQ Peg B (indicated by the decrease of the $\mathrm{A} / \mathrm{B}$ count ratio after $\sim 5 \mathrm{ksec}$ ) there is also some activity on EQ Peg A especially during the later phase of the observation.

From these ratios we calculated light curves for each individual component, which are shown in Fig. 7. Although the light curves consist of rather large time bins the main features visible in Fig. 5 are also present, i.e., a flare on EQ Peg B around $10 \mathrm{ksec}$ and the flaring activity on EQ Peg A towards the end of the observation. The rise in count rate associated with the flare activity on both components is nearly equally strong,
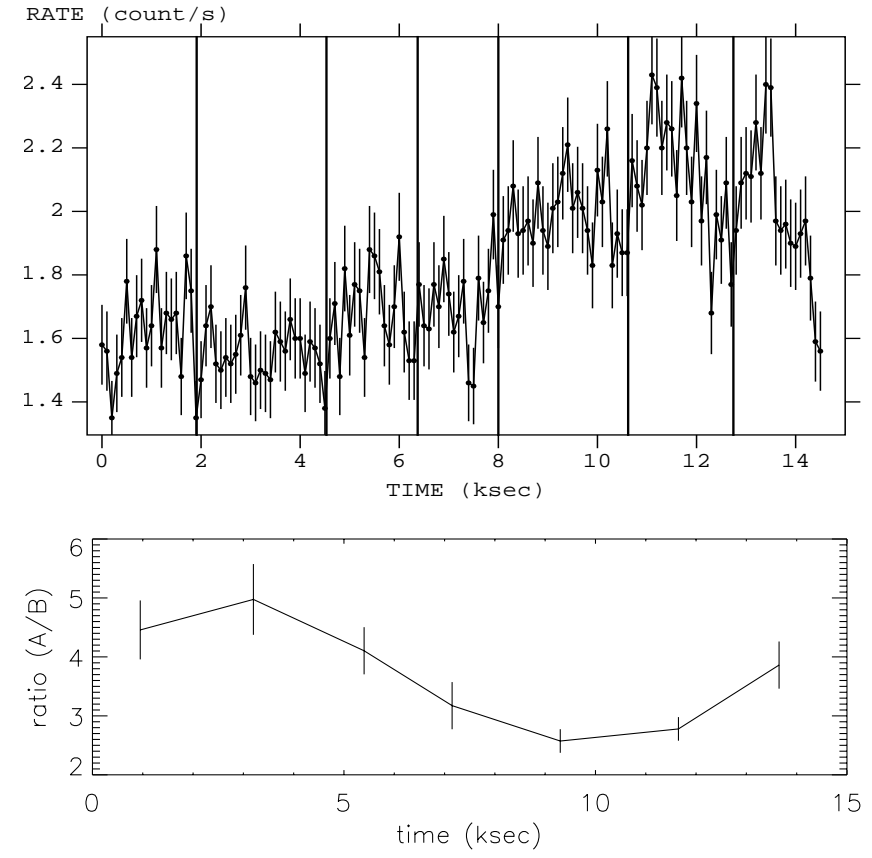

Fig. 6. Division of the MOS1 light curve into seven time intervals (top) and derived flux ratios (bottom).
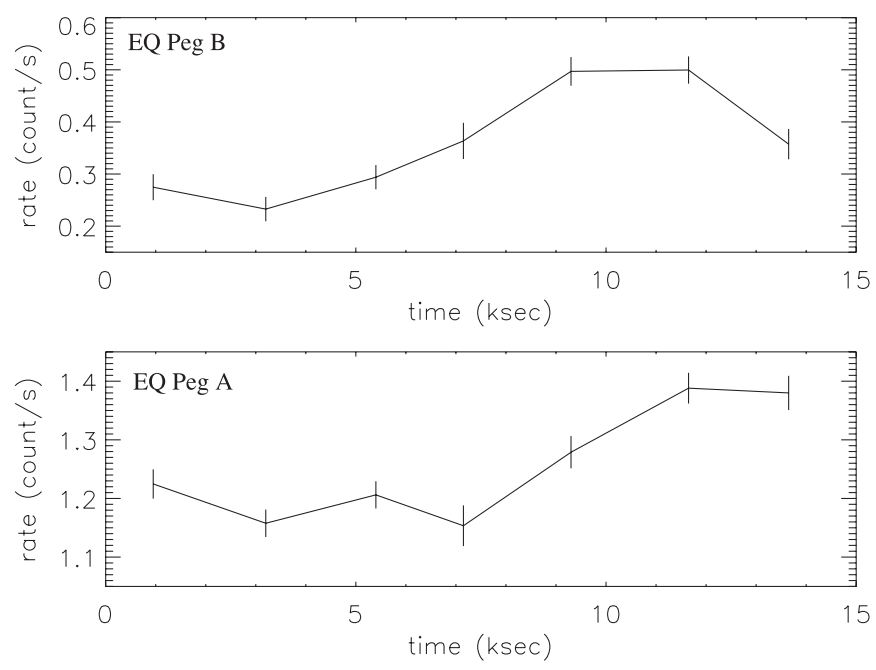

Fig. 7. Light curves of EQ Peg B (top) and A (bottom) calculated with the PSF fitting algorithm.

i.e., $\sim 0.2$ counts/s, however, the relative change in count rate is much higher on EQ Peg B.

\subsection{Development of the spectral hardness}

In order to quantify eventual changes in the physical conditions accompanied by rising count rates we calculate a spectral hardness ratio for the sum of both components in two energy bands, resp. $0.2-0.5 \mathrm{keV}$ (soft) and 1.0-10.0 keV (hard). The hardness ratio was calculated from $\mathrm{PN}$ data which were cleared for pile-up effects.

In Fig. 8 we show the spectral hardness ratio (hard/soft) for this observation binned every five minutes vs. the measured count rate. The hardness ratio increases during the times where 
HR (hard/soft)

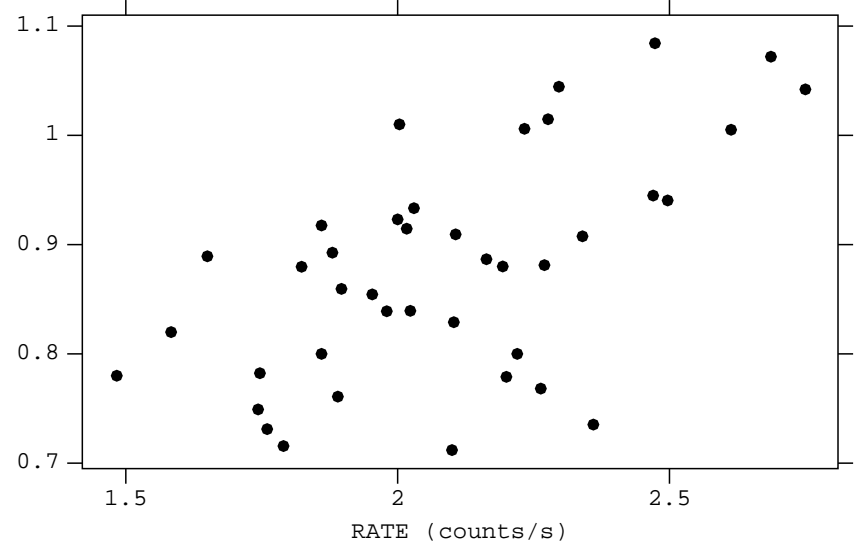

Fig. 8. Hardness ratio $(1-10 \mathrm{keV} / 0.2-0.5 \mathrm{keV})$ derived from $\mathrm{PN}$ data vs. count rate.

we also observe increases in count rate. We calculated a linear correlation coefficient and found a correlation probability of $99.9 \%$. We therefore interpret the simultaneous increase in count rate and hardness of the emission as flare heating of the coronal plasma. When comparing the calculated average hardness ratios during the time intervals defined in Sect. 3.2 as the quiescent phase and the flaring phase we find an increase in hardness by $12 \pm 2 \%$ from the quiescent phase into the flaring phase, whereas the increase during individual flares is substantially stronger.

The average X-ray luminosity between $0.2 \mathrm{keV}$ and $10.0 \mathrm{keV}$ was calculated to be $4.2 \times 10^{28} \mathrm{ergs} / \mathrm{s}$ from application of spectral models. We compared the X-ray luminosities obtained from a number of different reasonable models and find consistent results. In comparison with previous measurements the energy flux during this observation was $\sim 20 \%$ above the flux obtained with ROSAT (Hünsch et al. 1999) and 50\% below the values obtained with Einstein (Vaiana et al. 1981) in the respective energy bands.

\section{Summary and discussion}

Our analysis of EQ Peg is another example of how the highresolution X-ray telescopes Chandra and XMM-Newton allow one to resolve sources down to a unprecedented spatial resolution (for other examples see Stelzer \& Burwitz 2003 and Audard et al. 2003).

With the XMM-Newton observation of the EQ Peg system we were able to separate the two components for the first time in X-rays. Using a PSF model fit procedure we can reconstruct the source positions and show that both components are flaring X-ray emitters. On average, we found the A component brighter by a factor $\sim 3.5$ for the total observation.

During this observation a series of medium flares was detected. We were able to determine count ratios for EQ Peg A/B for the different phases of activity. During the early (quiescent) phase of the observation the emission is strongly dominated by EQ Peg A, which is a factor of 4-5 brighter than EQ Peg B. Comparison of the quiescent and active phases made it possible to associate most of the flaring with EQ Peg B, which nearly doubled it's X-ray brightness during the peak of the flare. The count ratio during the peak of the flare on EQ Peg B dropped to $\sim 2.5-3$. We also found evidence for flaring activity on EQ Peg A towards the end of the observation, consistent with previous findings that both stars exhibit flaring behavior (e.g., Rodonò 1978). In fact, the relative brightening during the flares is much stronger for EQ Peg B, but the absolute increase in flux is comparable for both stars. The energy released by these flares is obviously very similar, although the quiescent emission level is quite different. The flaring X-ray emission of the EQ Peg system shows the typical hardening in the spectral energy distribution as expected for stellar flares.

Acknowledgements. This work is based on observations obtained with XMM-Newton, an ESA science mission with instruments and contributions directly funded by ESA Member States and the USA (NASA). J.R. and J.-U.N. acknowledge support from DLR under 50OR0105.

\section{References}

Audard, M., Güdel, M., \& Skinner, S. L. 2003, ApJ, 589, 983

Bopp, B. W. 1974, MNRAS, 168, 255

Ehle, M., Breitfellner, M., Gonzales Riestra, M., et al. 2003, XMMNewton User's Handbook

Elvis, M., Plummer, D., Schachter J., et al. 1992, ApJS, 80, 257

Gagné, M., Valenti, J., Johns-Krull, C., et al. 1998, ASP Conf. Ser., 154,1484

Ghizzardi, S. 2001, EPIC-MCT-TN-011

Hünsch, M., Schmitt, J. H. M. M., Sterzik, M. F., et al. 1999, A\&AS, 135, 319

Katsova, M. M., Livshits, M. A., \& Schmitt, J. H. M. M. 2002, ASP Conf. Proc., 177, 515

Kirsch, M., \& EPIC Consortium, XMM-SOC-CAL-TN-0018

Owen, F. N., Bopp, B. W., Moffett, T. J., \& Lazor, F. J. 1972, ApL, 10, 37

Pallavicini, R., Kundu, M. R., \& Jackson, P. D. 1986, Lecture Notes in Physics, 254, 225

Perryman, M. A. C., Lindegren, L., Kovalevsky, J., et al. 1997, A\&A, 323, L49

Poletto, G., Pallavicini, R., \& Kopp, R. A. 1988, A\&A, 201, 93

Press, W. H., Teukolsky, S. A., Vetterling, W. T., et al. 1999, Numerical Recipes in C (Cambridge Univ. Press)

Rodonò, M. 1978, A\&A, 66, 175

Roques, P. E. 1954, PASP, 66, 256

Schmitt, J. H. M. M., Fleming, T. A., \& Giampapa, M. S. 1995, ApJ, 450,392

Schmitt, J. H. M. M., \& Liefke, C. 2003, A\&A, in press

Stelzer, B., \& Burwitz, V. 2003, A\&A, 402, 719

Topka, K., \& Marsh, K. A. 1982, ApJ, 254, 641

Vaiana, G. S., Cassinelli, J. P., Fabbiano, R., et al. 1981, ApJ, 244, 163 